\title{
Highly Sensitive Filter-Less Fluorescence Detection Method Using an Avalanche Photodiode
}

\author{
Masahiro Akiyama ${ }^{1}$, Kazuya Miyazawa ${ }^{1}$ and Kazuaki Sawada ${ }^{2}$ \\ 1. Department of Electrical and Electronic Engineering, National Institute of Technology, Nagano College, Nagano 381-8550, Japan \\ 2. Department of Electrical and Electronic Information Engineering, Toyohashi University of Technology, Aichi 441-8580, Japan
}

Received: February 03, 2016 / Accepted: March 08, 2016 / Published: April 30, 2016.

\begin{abstract}
Herein we report a highly sensitive filter-less fluorescence detection method using an APD (avalanche photodiode). Experimental measurements using the proposed APD-based highly sensitive fluorescence detection method exhibits the sensing capability to detect an excitation light and a fluorescence light without band pass filter or grating. The principle of this APD-based highly fluorescence detection method is used the varying multiplication ratio that is decided by wavelength. The wavelength controls running distance of photo-excited carrier by absorption coefficients, and this element decide multiplication ratio on fixed high electrical field. In fluorescence detection, they use two types of light: excitation light and fluorescence light. These lights have different wavelengths and make different multiplication ratio as well. Thus this method can separate two types of light easily by using multiplication ratios of APD without band pass filters/gratings. In this experiment, the excitation light is LED (light emitting diode) and fluorescence light occurs from FITC (fluorescein isothiocyanate) with ethanol. The FITC concentration changes from $0.1 \mu \mathrm{mol} / \mathrm{L}$ to $10 \mathrm{mmol} / \mathrm{L}$. In this measurement circuit, we employ APD (S2385), power supply voltage, and pico ampere current meter. As a result, these lights are correctly separated by using multiplication ratio with calculation at every concentration FITCs.
\end{abstract}

Key words: Avalanche photodiode, filter-less, fluorescence.

\section{Introduction}

Conventionally, fluorescent labels and an expensive fluorescent scanner are used for DNA (deoxyribonucleic acid) analysis $[1,2]$. The fluorescent scanner requires a special light source with a band pass filter [3, 4]. It is, in consequence, relatively bulky and low sensitivity by the filter. On the other hand, the biochemistry field has need of a straightforward space-saving and high sensitive fluorescence detection system [5-7].

To realize these requests, integration of the conventional system with low noise and high gain amplifier is required. The microchips possess following merits. Firstly, it is possible to miniaturize, so that it can incorporate with $\mu$-TAS (micro total analysis system) easily. Furthermore, if fluorescent

Corresponding author: Masahiro Akiyama, associate professor, research fields: photodetectors and imaging devices. information may be obtained, without using a fluorescent scanner and optical microscope, fluorescence analysis would be easier and be high sensitive than in a conventional system. In addition, APDs (avalanche photodiodes) fabricated in CMOS (complementary metal oxide semiconductor) process make low noise and have amplifier gain [8]. Thus we propose APD-based highly sensitive fluorescence detection method. There is another merit: excitation light intensity with getting fluorescence light intensity detects at same time. The detecting excitation light intensity improves accuracy of fluorescence light intensity. For the first time, in this paper, we report an APD-based filter-less fluorescence detection method.

\section{Method}

Our proposed method uses APD (S2385). APDs have multiplication ratio having incident light wavelength dependent properties. This means, when 
single light (excitation light or fluorescence light) radiates to the APD, the multiplication ratio $\left(M_{E}\right.$ or $M_{F}$ ) is controlled by the wavelength with $73 \mathrm{~V}$ power supply voltage. $M_{E}$ and $M_{F}$ are calculated as follows:

$$
M_{E}=\left(\frac{I p_{E}(73 \mathrm{~V})}{I p_{E}(50 \mathrm{~V})}\right), \quad M_{F}=\left(\frac{I p_{F}(73 \mathrm{~V})}{I p_{F}(50 \mathrm{~V})}\right)
$$

$I p_{E}(73 \mathrm{~V})$ and $I p_{E}(50 \mathrm{~V})$ are photocurrent of APD by excitation light with $73 \mathrm{~V}$ and $50 \mathrm{~V}, \operatorname{Ip}_{F}(73 \mathrm{~V})$ and $I p_{F}(50 \mathrm{~V})$ are photocurrent of APD by fluorescence light with $73 \mathrm{~V}$ and $50 \mathrm{~V}$. These photocurrent subtract dark current $\left(I_{d}(50 \mathrm{~V}), I_{d}(73 \mathrm{~V})\right)$ for accurate calculation. These photocurrents and dark current are measured by pico ampere meter in measurement circuit of Fig. 1. When mixed light (excitation light and fluorescent light) radiates to the APD, the mixed multiplication ratio $\left(M_{\text {Mixed }}\right)$ is controlled by mixed ratios $\left(X_{E}, X_{F}\right)$ of these lights (excitation light and fluorescent light) with $73 \mathrm{~V}$ power supply voltage. The $M_{\text {Mixed }}$ is calculated as follows:

$$
\begin{gathered}
M_{\text {Mixed }}=\frac{I p_{\text {Mixed }}(73 \mathrm{~V})}{I p_{\text {Mixed }}(50 \mathrm{~V})}=\frac{I p_{E}(73 \mathrm{~V})+I p_{F}(73 \mathrm{~V})}{I p_{E}(50 \mathrm{~V})+I p_{F}(50 \mathrm{~V})} \\
=\frac{M_{E} \times I p_{E}(50 \mathrm{~V})+M_{F} \times I p_{F}(50 \mathrm{~V})}{I p_{E}(50 \mathrm{~V})+I p_{F}(50 \mathrm{~V})} \\
=\frac{M_{E} \times I p_{E}(50 \mathrm{~V})}{I p_{E}(50 \mathrm{~V})+I p_{F}(50 \mathrm{~V})}+\frac{M_{F} \times I p_{F}(50 \mathrm{~V})}{I p_{E}(50 \mathrm{~V})+I p_{F}(50 \mathrm{~V})} \\
=M_{E} \times X_{E}+M_{F} \times X_{F}
\end{gathered}
$$

And $X_{E}, X_{F}$ have Eq. (3):
$X_{E}+X_{F}=\frac{I p_{E}(50 \mathrm{~V})}{I p_{E}(50 \mathrm{~V})+I p_{F}(50 \mathrm{~V})}+\frac{I p_{B}(50 \mathrm{~V})}{I p_{E}(50 \mathrm{~V})+I p_{E}(50 \mathrm{~V})}=1$

Then, $X_{E}, X_{F}$ calculate as follows:

$$
X_{E}=\frac{M_{\text {Mixed }}-M_{F}}{M_{E}-M_{F}}, X_{F}=\frac{M_{\text {Mixed }}-M_{E}}{M_{F}-M_{E}}
$$

The quantum efficiency of excitation light $\left(\eta_{E}\right)$ and fluorescent light $\left(\eta_{F}\right)$ with the APD are 0.63 and 0.73 , respectively. The $\eta_{E}$ and $\eta_{F}$ are the number of EHPs (electron-hole pairs) generated from each incident photon:

$$
\begin{gathered}
\eta_{E}=\left(\frac{X_{E} \times I p_{\text {Mixed }}(50 \mathrm{~V})}{q}\right) \cdot\left(\frac{W_{E}}{h \frac{c}{\lambda_{E}}}\right)^{-1} \\
\eta_{F}=\left(\frac{X_{F} \times I p_{\text {Mixed }}(50 \mathrm{~V})}{q}\right) \cdot\left(\frac{W_{F}}{h \frac{c}{\lambda_{F}}}\right)^{-1}
\end{gathered}
$$

where, $I p_{\text {Mixed }}(50 \mathrm{~V})$ is the photogenerated current from the absorption of incident optical power $W_{E}$ and $W_{F}$ at their wavelength $\lambda_{E}$ (corresponding to a photon energy $h c / \lambda_{E}$ ) and $\lambda_{F}$ (corresponding to a photon energy $\left.h c / \lambda_{F}\right)$. Then $W_{E}$ and $W_{F}$ are expressed as follows:

$$
\begin{gathered}
W_{E}=\left(\frac{X_{E} \times I p_{\text {Mixed }}(50 \mathrm{~V})}{q}\right) \cdot\left(\frac{\eta_{E}}{h \frac{c}{\lambda_{E}}}\right)^{-1} \\
W_{F}=\left(\frac{X_{F} \times I p_{\text {Mixed }}(50 \mathrm{~V})}{q}\right) \cdot\left(\frac{\eta_{F}}{h \frac{c}{\lambda_{F}}}\right)^{-1}
\end{gathered}
$$

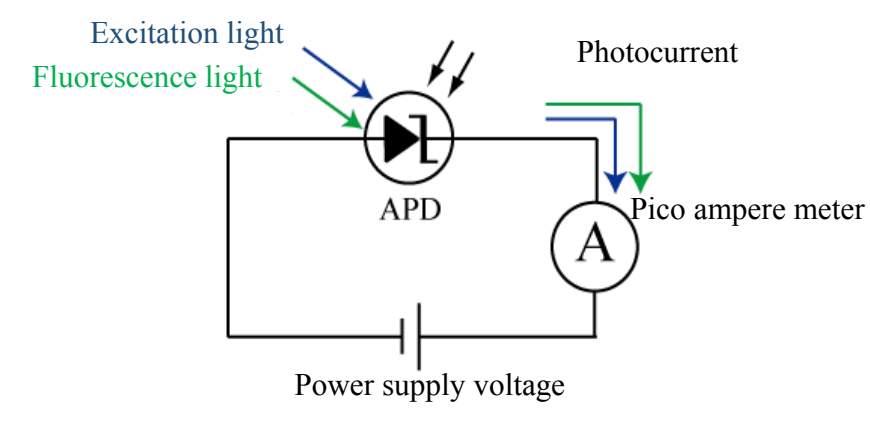

Fig. 1 Measurement circuit for measuring photocurrent and dark current. 
From these equations, we can find $W_{E}$ and $W_{F}$ from measurement $I p_{\text {Mixed }}(50 \mathrm{~V})$ and $I p_{\text {Mixed }}(73 \mathrm{~V})$.

\section{Experiment}

Figs. $2 \mathrm{a}$ and $2 \mathrm{~b}$ show measurement system from top side and cross side respectively. The LED is used as an excitation light that have $470 \mathrm{~nm}$ wavelength peak. The palette has solution mixed with ethanol and FITC (fluorescein isothiocyanate), and that total amount becomes $300 \mu \mathrm{L}$. We prepare seven patterns solutions for testing $W_{F}$ detection by APD without filters. These FITC concentrations are $0 \mu \mathrm{mol} / \mathrm{L}$ (Solution 1), $0.1 \mu \mathrm{mol} / \mathrm{L}$ (Solution 2), $1 \mu \mathrm{mol} / \mathrm{L}$ (Solution 3), $10 \mu \mathrm{mol} / \mathrm{L}$ (Solution 4), $0.1 \mathrm{mmol} / \mathrm{L}$ (Solution 5), $1 \mathrm{mmol} / \mathrm{L}$ (Solution 6), $10 \mathrm{mmol} / \mathrm{L}$ (Solution 7), respectively. Set APD which is connected to power supply voltage and pico ampere meter under the palette in order to detect mixed light $\left(W_{E}, W_{F}\right)$. After the measurement, spectroscope place under the palette instead of APD for comparing APD output $\left(W_{E}, W_{F}\right)$ and spectroscope output.

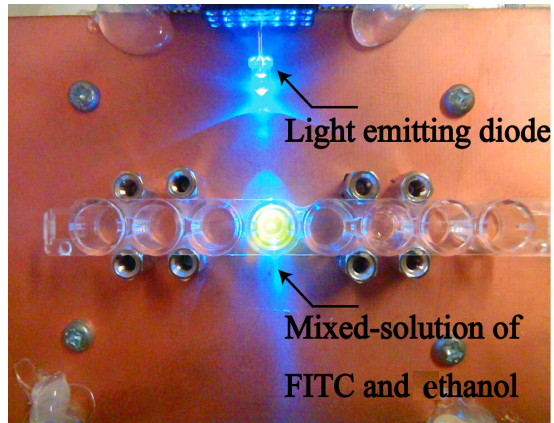

(a)

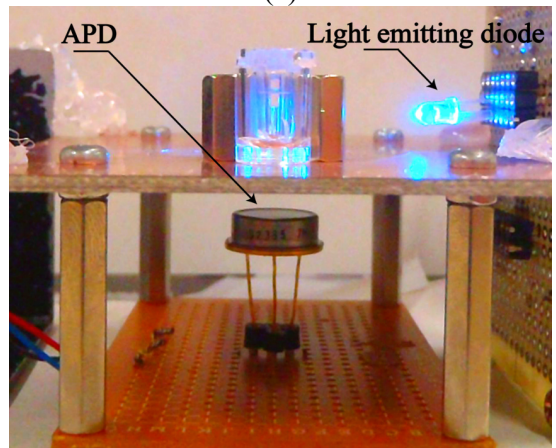

(b)

Fig. 2 Measurement system from (a) top side and (b) cross side under the palette instead of APD for comparing APD output $\left(W_{E}, W_{F}\right)$ and spectroscope output.

\section{Measurement Flow}

Measurement flow is separated a basic measurement and a target measurement. At first, we try to measure them using basic measurement as follows:

(1). Measure dark current $\left(I_{d}(50 \mathrm{~V}), I_{d}(73 \mathrm{~V})\right)$ of APD on measurement circuit (Fig. 3).

(2). Measure photocurrent $\left(\operatorname{Ip}_{E}(50 \mathrm{~V}), \operatorname{Ip}_{E}(73 \mathrm{~V})\right)$ by excitation light (Fig. 4). Then calculate multiplication ratio $\left(M_{E}\right)$ from $I p_{E}(50 \mathrm{~V})$ and $I p_{E}(73 \mathrm{~V})$ using Eq. (1).

(3). Measure photocurrent (I M $_{\text {Mixed }}(50 \mathrm{~V})$, I Mixed $_{\text {Mix }}$ $(73 \mathrm{~V}))$ by mixed light. Then, calculate photocurrent $\left(I p_{F}(50 \mathrm{~V}), I p_{F}(73 \mathrm{~V})\right)$ from differential $I p_{\text {Mixed }}(73$ V) to $I p_{E}(73 \mathrm{~V})$ (Fig. 5).

(4). Calculate multiplication ratio $\left(M_{F}(73 \mathrm{~V})\right)$ from $I p_{F}(50 \mathrm{~V}), I p_{F}(73 \mathrm{~V})$ using Eq. (1).

After finishing this basic measurement, target measurement is performed as follows:

(1). Measure mixed photocurrent (I Mixed $_{\text {Mix }}(50 \mathrm{~V})$, Ip $p_{\text {Mixed }}(73 \mathrm{~V})$ ) by mixed light (Fig. 6).

(2). Calculate multiplication ratio $\left(M_{\text {Mixed }}\right)$ using Eq. (2).

(3). Calculate mixed ratio $\left(X_{E}, X_{F}\right)$ using Eq. (4).

(4). Calculate light intensity $\left(W_{E}, W_{F}\right)$ using Eq. (6). Through these calculations, $W_{E}$ and $W_{F}$ are detected.

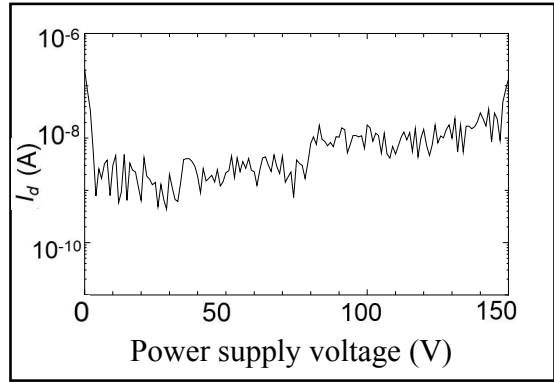

Fig. 3 Dark current.

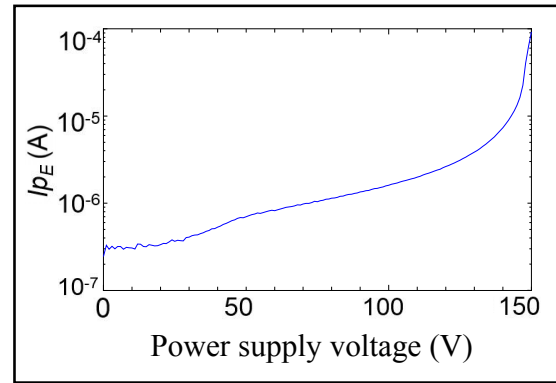

Fig. 4 Photocurrent by $W_{E}$. 


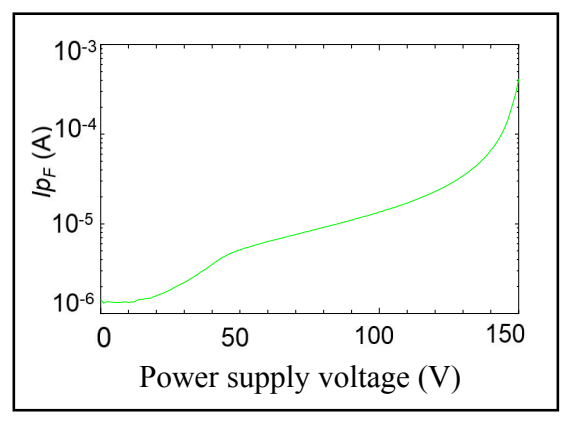

Fig. 5 Photocurrent by $W_{F}$.

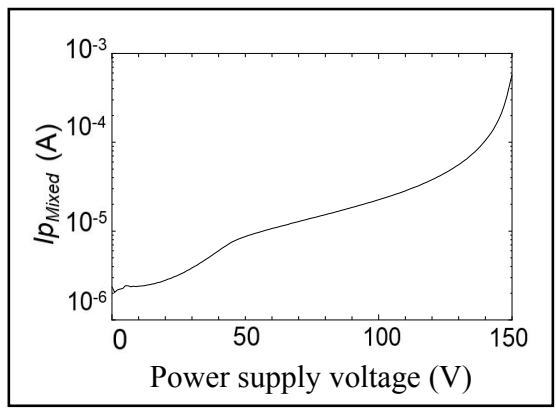

Fig. 6 Photocurrent by $W_{E}+W_{F}$.

\section{Results}

We acquired $W_{E}$ and $W_{F}$ from calculated APD output and light intensity spectrum from spectroscope with this measurement system (Fig. 2a). The light intensity spectrum has two peaks, one is excitation light peak and the other is fluorescence light peak. We call this amount of the peaks to $P_{E}$ and $P_{F}$, respectively. Fig. 7 shows light intensity spectrum that has $470 \mathrm{~nm}$ peak $\left(P_{E}\right)$ with Solution 1 by excitation light. There is not any other peak from fluorescence light because FITC concentration is $0 \mathrm{~mol} / \mathrm{L}$. In this time, $W_{E}$ and $W_{F}$ are, calculated APD output from Eq. (6), $4.28 \mu \mathrm{W}$ and $0.0 \mathrm{~W}$, respectively. Fig. 7a shows light intensity spectrum with Solution 2. There is one big peak and several small peaks; big one is $P_{E}$, several peaks are made by fluorescence light and peak has not yet cleared. The $W_{E}$ and $W_{F}$, detected by calculated APD output are $3.17 \mu \mathrm{W}$ and $0.09 \mu \mathrm{W}$, respectively. This means that, this method has more highly sensitive detection compared with the spectroscope on this system. Fig. 7b shows light intensity spectrum with Solution 3 . There are two big peaks: $P_{E}$ and $P_{F}$. This $P_{F}$ is detected bigger than $P_{F}$ of Fig. 7c. The $W_{E}$ and $W_{F}$ detected by calculated APD output are $3.73 \mu \mathrm{W}$ and $1.16 \mu \mathrm{W}$, respectively. Fig. $7 \mathrm{~d}$ shows light intensity spectrum with Solution 4 . The $P_{F}$ becomes bigger than $P_{E}$, and $W_{F}$ becomes bigger than $W_{E}$. The spectroscope and APD provide similar output. Figs. $7 \mathrm{e}$ and $7 f$ show light intensity spectrum with Solutions 5 and 6, respectively. The $P_{E}$ is decreasing with FITC concentration, while the $P_{F}$ is increasing with FITC concentration. Fig. $7 \mathrm{~g}$ shows light intensity spectrum with Solution 7. $P_{E}$ is very small compared with $P_{F}$. This shows $P_{E}$ is difficult to separate other small peaks which are created by big fluorescence light. On the other hand, $W_{E}$ and $W_{F}$ detected by calculating APD output are $3.45 \mu \mathrm{W}$ and $24.8 \mu \mathrm{W}$, respectively. This result means that, our method has more highly dynamic range compared with the spectroscope on the system.

Fig. 8 shows $W_{E}$ and $W_{F}$ characteristics of FITC concentrations: $W_{F}$ is increased with the concentration, while the $W_{E}$ is not depend on the concentrations. As a result, this method can separately sense $W_{E}$ and $W_{F}$ in any FITC concentrations. 


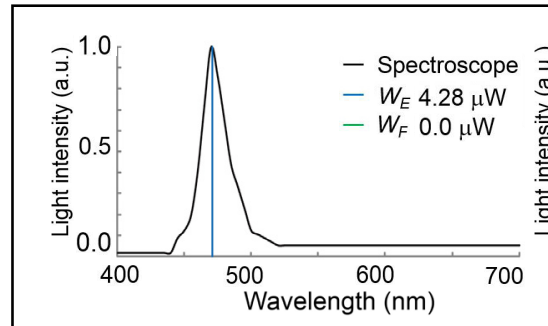

(a)

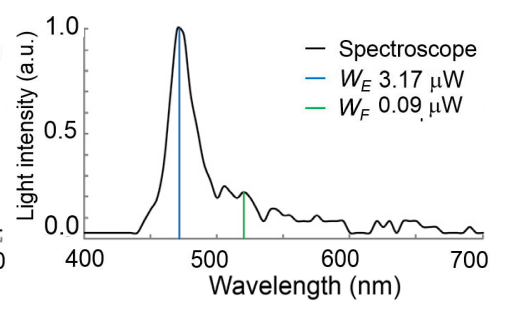

(b)

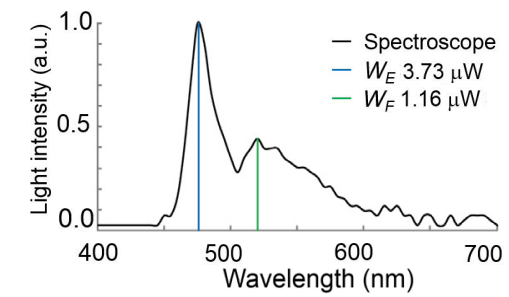

(c)

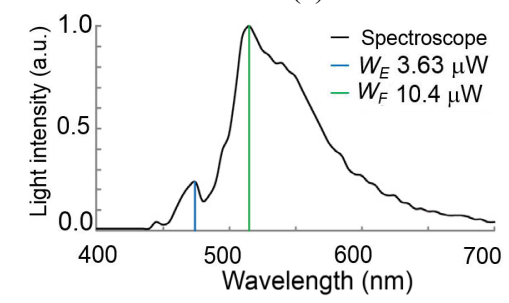

(e)

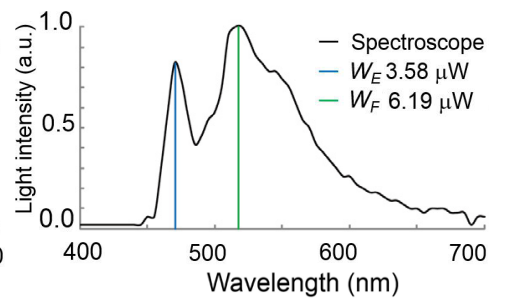

(d)

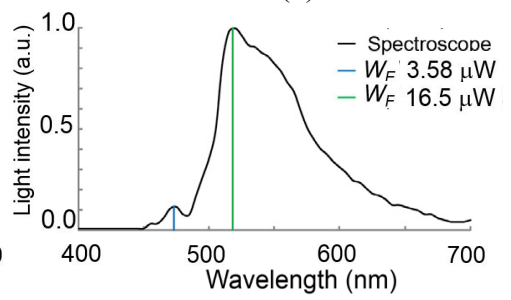

(f)

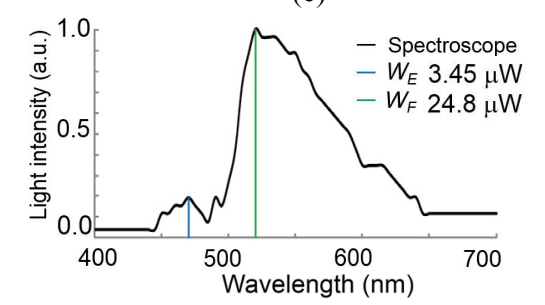

(g)

Fig. 7 Light intensity spectrum with (a) Solution 1, (b) Solution 2, (c) Solution 3, (d) Solution 4, (e) Solution 5, (f) Solution 6, (g) Solution 7.

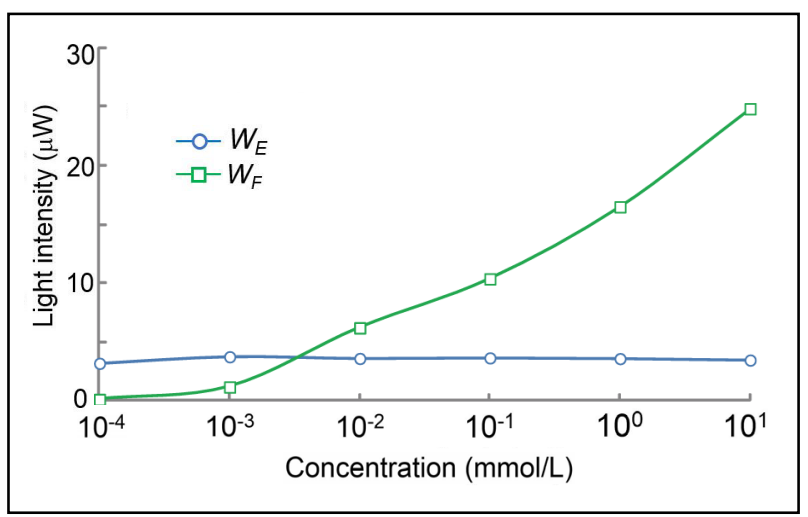

Fig. $8 W_{E}, W_{F}$ characteristics of FITC concentrations light.

\section{Conclusions}

These two light intensity values can be calculated from mixed multiplication ratio. Through these results, we can realize filter less $W_{F}$ detection by APD, and we can achieve to make $W_{F}$ detection system minimized on a chip with high gain, low noise and high dynamic range characteristics. Moreover, this method obtains both light intensity $\left(W_{E}, W_{F}\right)$ at the same time. It also has a significant merit for improving accuracy of $W_{F}$ value, because $W_{E}$ is clearly effected by $W_{F}$ value.

\section{References}

[1] Jun, L., Hongfei, Y., Kemin, W., Weihong, T., and Xingwang, Z. 2007. "Hairpin Fluorescence DNA Probe for Real-Time Monitoring of DNA Methylation." Analytical Chemistry 79 (3): 1050-6.

[2] Xavier, L. G., Christian, S., Nicole, D., Gregor, J., and Marc, S. 2012. "Highly Fluorescent Silver Nanoclusters 
Stabilized by Glutathione: A Promising Fluorescent Label for Bioimaging." Nano. Research 5 (6): 379-87.

[3] John, E., Hobbie, R., Daley, J., and Jasper, S. 1977. "Use of Nuclepore Filters for Counting Bacteria by Fluorescence Microscopy." Applied and Environmental Microbiology 33 (5): 1225-8.

[4] Li, S., Linxiao, Y., Florian, S., Radian, P., Vanessa, T., Michael, B., Dagmar, G., and Ulrich, G. N. 2012. "Microwave-Assisted Rapid Synthesis of Luminescent Gold Nanoclusters for Sensing $\mathrm{Hg}^{2+}$ in Living Cells Using Fluorescence Imaging." Nanoscale 14 (4): 4155-60.

[5] Yuki, M., Kazuaki, S., Hidekuni, T., and Makoto, I. 2006. "The Fabrication of Filter-Less Fluorescence Detection Sensor Array Using CMOS Image Sensor Technique."
Sensors and Actuators A 12 (1): 66-70.

[6] Christin, B., Jens, K., Heinrich, G., and Hubert, L. 2014. "Large Field of View MEMS-Based Confocal Laser Scanning Microscope for Fluorescence Imaging." Optik 125 (2): 876-82.

[7] Marcela, M. W., Cheng, W., Chuan-De, W., and Wenbin, L. 2012. "A Chiral Porous Metal? Organic Framework for Highly Sensitive and Enantioselective Fluorescence Sensing of Amino Alcohols." Journal of the American Chemical Society 134 (22): 9050-3.

[8] Myung-Jae, L., Holger, R., and Woo-Young, C. 2012. "Effects of Guard-Ring Structures on the Performance of Silicon Avalanche Photodetectors Fabricated with Standard CMOS Technology." IEEE Electron Device Letters 33 (1): 80-2. 